\title{
Isabella Sudano \\ Cholesterin und Atherosklerose - landläufige Irrtümer und wissenschaftliche Erkenntnisse
}

Über keinen anderen kardiovaskulären Risikofaktor gibt so viele Kontroversen wie über das Cholesterin: Gibt es «gutes» und «schlechtes» Cholesterin? Ist erhöhtes Cholesterin ein Risikofaktor für Atherothrombose und kardiovaskuläre Erkrankungen? Kann ich meine Cholesterinwerte ohne Medikamente normalisieren?

Cholesterin: wie es gemessen wird und welches die Zielwerte sind

Was für die Beurteilung des kardiovaskulären Risikos wichtig ist, ist die Messung des Cholesterins im Blut. Da Fette, Cholesterin und Fettbegleitstoffe nicht wasserlöslich sind, stellt ihnen der Körper für den Transport im Blut Trägerproteine, sogenannte Lipoproteine, zur Verfügung. ${ }^{1}$ Es gibt verschiedene Lipoproteine, wobei vor allem zwei Arten bekannt sind: Das LDL (low density lipoprotein) und das HDL (high density lipoprotein). ${ }^{2}$ Normalerweise werden das Gesamt-, das LDL- und das HDL-Cholesterin sowie die Triglyzeride im Blut bestimmt. Erhöhte LDL-Werte sind unerwünscht,

$1 \quad$ Alan T. Remaley, Thomas D. Dayspring, G. Russel Warnick: Lipids, lipoproteins, apolipoproteins, and other cardiovascular risk factors, in: Nader Rifai, Andrea R. Horvath, Carl T. Wittwer (eds.): Tietz Textbook of Clinical Chemistry and Molecular Diagnostics, St. Louis 2018, 539-603.

2 Ebd. 
weil sie die Atherosklerose fördern. Bei gesunden Personen ist ein LDL $<3 \mathrm{mmol} / \mathrm{L}$, bei Patienten, die bereits ein kardiovaskuläres Ereignis erlitten, ein LDL $<1.8 \mathrm{mmol} / \mathrm{L}$ wünschenswert. Ein HDL $<1 \mathrm{mmol} / \mathrm{L}$ zählt als kardiovaskulärer Risikofaktor. ${ }^{3}$

Triglyzeride werden vom Körper hergestellt aus überschüssigem Zucker, das heisst aus Kohlehydraten, die wir zu uns nehmen. Die Cholesterinwerte können durch angeborene Mängel oder durch falsche Ernährung, Erkrankungen und bestimmte Medikamente ansteigen. $\mathrm{Ob}$ erhöhte Triglyzeride die Gefässe direkt schädigen können, ist umstritten. ${ }^{4}$ Ein zu hoher Triglyzeridspiegel ist aber ein Zeichen für einen gestörten Fettstoffwechsel und kann zur Entzündung der Bauchspeicheldrüse führen.

Entgegen früheren Meinungen kann das Plasma-Lipidprofil in den meisten Fällen auch nach Nahrungseinnahme erstellt werden. In bestimmten Situationen wird jedoch eine Nüchtern-Blutentnahme empfohlen: bei Nicht-Nüchtern Triglyzeriden $>5 \mathrm{mmol} / \mathrm{l}$, bei bekannter Hypertriglyzeridämie oder bei Hypertriglyzeridämieinduzierter Pankreatitis, vor Beginn einer medikamentösen Therapie mit schwerer Hypertriglyzeridämie als möglicher Nebenwirkung oder wenn zusätzliche Analysen gefordert sind, die nüchtern zu bestimmen sind (beispielsweise Nüchtern-Glukose, therapeutisches Medikamenten-Monitoring). ${ }^{5}$

Ebd.; http://www.agla.ch

Zeljko Reiner: Hypertriglyceridaemia and risk of coronary artery disease, in: Nature Reviews Cardiology 14 (2017) 401-411.

Børge G. Nordestgaard et al.: Fasting Is Not Routinely Required for Determination of a Lipid Profile. Clinical and Laboratory Implications Including Flagging at Desirable Concentration Cutpoints. A Joint Consensus Statement from the European Atherosclerosis Society and European Federation of Clinical Chemistry and Laboratory Medicine, in: Clinical Chemistry 62 (2016) 930-946. 


\section{Cholesterin und Atherothrombose}

Cholesterin wird vom Körper selbst - vor allem in der Leber gebildet und erfüllt wichtige Aufgaben. Unter anderem wird Cholesterin zum Aufbau der Zellen, zum Beispiel für die Zellmembranen, gebraucht und ist an verschiedenen Stoffwechselvorgängen des Gehirns und an der Produktion von Hormonen beteiligt. ${ }^{6}$ Jede Zelle baut Cholesterin für den eigenen Gebrauch auf; die Reduktion des LDL-Cholesterins im Blut beeinflusst die intrazelluläre Konzentration des Cholesterins nicht. ${ }^{7}$

$\mathrm{Zu}$ viel Cholesterin war in der Vergangenheit vermutlich vorteilhaft: Die Lebenserwartung war so kurz, dass die Atherosklerose gar kein Problem war und Menschen mit hohen Cholesterinwerten hatten Vorteile, wenn ihnen für längere Zeit keine oder nur wenige Nahrungsmittel zur Verfügung standen.

Die Plausibilität eines Zusammenhanges zwischen LDL-Cholesterin und Atherosklerose ist eindeutig gegeben. Das LDL-Cholesterin ist direkt an der Entstehung und dem Fortschreiten der Atherosklerose beteiligt. $^{8}$ Eine experimentell induzierte Erhöhung des Plasma-LDL und anderer ApoB-haltiger Lipoproteine führe in allen untersuchten Säugetierspezies zur Atherosklerose. ${ }^{9}$

In einem Artikel wurden die Daten von über 200 Studien mit mehr als zwei Millionen Teilnehmenden sowie mehr als 150000 kardiovaskulären Ereignissen untersucht. Übereinstimmend liess sich eine dosisabhängige, lineare Assoziation zwischen dem absoluten

6 Christopher J. Packard, James Shepherd: The pathophysiology of cholesterol metabolism in man, in: Klinische Wochenschift 63 (1985) 344-351; Eliahu August, Kim H. Parker, Mauricio Barahona: A dynamical model of lipoprotein metabolism, in: Bulletin of Mathematical Biology 69 (2007) 1233-1254.

$7 \quad$ E. August usw., a.a.O.

8 A. T. Remaley, T. D. Dayspring, G. R. Warnick: Lipids, lipoproteins, apolipoproteins.

9 Godfrey S. Getz, Catherine A. Reardon: Animal models of atherosclerosis, in: Arteriosclerosis, Thrombosis, and Vascular Biology 32 (2012) 11041115. 
Ausmass einer LDL-Exposition und der Höhe des Atheroskleroserisikos belegen. ${ }^{10}$ Ein schlagkräftiges Argument für einen kausalen Zusammenhang zwischen LDL-Cholesterin und Atherosklerose sind die kardiovaskulären Auswirkungen vererbbarer Stoffwechselerkrankungen wie der familiären Hypercholesterinämie. ${ }^{11}$

Menschen, die eine Mutation in einem der am Cholesterinstoffwechsel beteiligten Gene aufweisen, haben deutlich erhöhte LDLCholesterin-Werte. Damit einhergehend leiden die betroffenen Personen - wenn die Erkrankung unerkannt und unbehandelt bleibt frühzeitig an kardiovaskulären Erkrankungen. Demgegenüber haben Personen mit Loss-of-Function-Mutationen im PCSK9-Gen, die mit sehr niedrigen LDL-Cholesterin-Konzentrationen einhergehen, ein auffällig niedriges kardiovaskuläres Risiko. ${ }^{12}$

Therapeutische Ansätze, die genau an diesen Stellschrauben angreifen, führen wiederum zu einer merklichen Reduktion kardiovaskulärer Ereignisse. ${ }^{13}$ Die dadurch erreichte Risikosenkung ist proportional zu der LDL-Cholesterin-Senkung, die durch die Medikation erreicht wird. Also je tiefer das LDL-Cholesterin gesenkt wird, desto niedriger ist das kardiovaskuläre Risiko. Eine solche Dosisabhängigkeit ist ein weiteres Kriterium, das für eine Kausalität eines Zusammenhanges erfüllt sein sollte. ${ }^{14}$

10 Brian A. Ference et al.: Low-density lipoproteins cause atherosclerotic cardiovascular disease. 1. Evidence from genetic, epidemiologic, and clinical studies. A consensus statement from the European Atherosclerosis Society Consensus Panel, in: European Heart Journal 38 (2017) 2459-2472.

11 Ebd.

12 Ebd.

13 Cholesterol Treatment Trialists Collaboration, Borislava Mihaylova, Jonathan Emberson et al.: The effects of lowering LDL cholesterol with statin therapy in people at low risk of vascular disease: meta-analysis of individual data from 27 randomised trials, in: The Lancet 380 (2012) 581590.

14 Ebd.; Colin Baigent, Anthony Keech, Patricia M. Kearney et al.: Efficacy and safety of cholesterol-lowering treatment: prospective meta-analysis of data from 90,056 participants in 14 randomised trials of statins, in: The Lancet 366 (2005) 1267-1278. 
So liess sich in einer Metaanalyse mit 26 Statin-Studien das kardiovaskuläre Risiko der fast 170000 Teilnehmer während einer fünfjährigen Statin-Behandlung proportional um $22 \%$ pro mmol / L erreichte LDL-Cholesterin-Reduktion senken. ${ }^{15}$ Überzeugend ist auch die Wirksamkeit der neuen PCSK9-Inhibitoren, welche die LDL-Cholesterin-Spiegel auf ein sehr tiefes Niveau senken. Bereits nach einer mittleren Behandlungszeit von 2.2 Jahren ging die Rate an Herzinfarkten und Schlaganfällen in der FOURIER_- ${ }^{16}$ sowie in den Odyssey-Studie ${ }^{17}$ um ca. $20 \%$ zurück.

\section{Kumulativer schädlicher Effekt des LDL-Cholesterins}

Das LDL-Cholesterin stellte sich als stärkster unabhängiger modifizierbarer Risikofaktor für die Atherosklerose heraus, und ein linearer Zusammenhang zwischen dem absoluten Ausmass der LDL-Cholesterin-Exposition und der Höhe des kardiovaskulären Risikos ist über längere Zeit zu beobachten. ${ }^{18}$ Darüber hinaus belegen

B. A. Ference et al.: Low-density lipoproteins cause atherosclerotic cardiovascular disease.

Robert P. Giugliano, Terje R. Pedersen, Jeong-Gun Park et al.: Clinical efficacy and safety of achieving very low LDL-cholesterol concentrations with the PCSK9 inhibitor evolocumab: a prespecified secondary analysis of the FOURIER trial, in: The Lancet 390 (2017) 1962-1971; Marc S. Sabatine, Lawrence A. Leiter, Stephen D. Wiviott et al.: Cardiovascular safety and efficacy of the PCSK9 inhibitor evolocumab in patients with and without diabetes and the effect of evolocumab on glycaemia and risk of new-onset diabetes: a prespecified analysis of the FOURIER randomised controlled trial, in: The Lancet Diabetes \& Endocrinology 5 (2017) 941950.

Michael Szarek, Harvey D. White, Gregory G. Schwartz et al.: Alirocumab Reduces Total Nonfatal Cardiovascular and Fatal Events: The ODYSSEY OUTCOMES Trial, in: Journal of the American College of Cardiology 73 (2019) 387-396.

Brian A. Ference, Ian Graham, Lale Tokgozoglu, Alberico L. Catapano: Impact of Lipids on Cardiovascular Health: JACC Health Promotion Series, in: Journal of the American College of Cardiology 72 (2018) 11411156. 
Studien mit Mendelscher Randomisierung einen kumulativen, also mit der Zeit zunehmenden schädlichen Effekt einer dauerhaften (hier genetisch bedingten) LDL-Cholesterin-Erhöhung. ${ }^{19}$

Alles in allem gebe es somit eine eindeutige und konsistente Evidenz, dass das LDL-Cholesterin nicht nur einen Biomarker darstelle, sondern ursächlich an der Pathophysiologie der Atherosklerose beteiligt sei. In Anbetracht des kumulativen schädlichen Effektes einer dauerhaften LDL-Cholesterin-Erhöhung könnte es sinnvoll sein, bei Hochrisikopatienten das LDL-Cholesterin sogar noch früher als bisher empfohlen therapeutisch zu senken.

Rolle der Ernährung und von Lebensstil-Veränderungen als Therapie einer Dyslipidämie

Die Cholesterinwerte im Blut werden durch verschiedene Faktoren beeinflusst: Alter, Geschlecht, Essgewohnheiten, Menopause, gewisse genetische Veranlagungen sowie gewisse Krankheiten (wie beispielsweise Schilddrüsenüberfunktion oder Diabetes). Zum grössten Teil wird das Cholesterin im Körper selbst produziert, vor allem in der Leber. Diese körpereigene Bildung reduziert sich je nach Cholesterinzufuhr über die Nahrung. So besteht zwischen Eigenproduktion und Aufnahme über die Nahrung ein gewisses Gleichgewicht. Ist zu viel Cholesterin im Blut, gelangt es zurück in die Leber und wird ersorgt. Bei genetischer Hypercholesterinämie ist die Produktion nicht mehr korrekt reguliert oder ist die Aufnahme von Cholesterin zurück in die Leber gestört. Auf diese Weise steigt die Cholesterinmenge im Blut über ein wünschenswertes Mass an.

Durch Ernährungsumstellung und mehr Bewegung kann häufig eine Verbesserung der Werte erreicht werden. Doch immer weniger Menschen gelangen mit solchen Massnahmen zu Wunschwerten: Die Nahrungscholesterin-Zufuhr beeinflusst den Cholesterinspiegel 
nur sehr gering. Bei ausgewogener Ernährung beträgt der Anteil des Plasma-LDL, der durch die Nahrung beeinflusst werden kann, etwa $10 \% .{ }^{20}$ So ist es für viele Personen nicht möglich, mit nicht pharmakologischen Therapien die empfohlenen Zielwerte zu erreichen. Anders als bei Cholesterin können die Triglyzeride durch Ernährungsumstellung deutlich gesenkt werden. Die Gewohnheit, das erhöhte Cholesterin mit Eier- oder Fleischkonsum zu assoziieren, ist deshalb nicht korrekt. ${ }^{21}$ Es gibt Studien, die zeigen, dass eine vegane Ernährung das Plasma-LDL nicht wesentlich senkt. ${ }^{22}$

Obwohl das LDL-Cholesterin numerisch nicht wesentlich durch Ernährungsumstellung sinkt, beeinflusst das, was wir essen, die Qualität des LDL-Cholesterins sehr stark. Früchte und Gemüse sowie andere Nahrungsmittel, die reich in Antioxidantien sind, verhindern beispielsweise die Oxidation des LDL-Cholesterins und spielen deshalb in der Prävention der Atherothrombose eine grosse Rolle. ${ }^{23} \mathrm{Zu}$ den nicht pharmakologischen Therapien der Dyslipidämie gehören regelmässige Bewegung mit mindestens 30 bis 45 Minuten aerobem Training dreimal wöchentlich, Gewichtsabnahme bei Übergewicht und Abstinenz von Tabak-Konsum. ${ }^{24}$

$20 \quad$ http://www.sge-ssn.ch

21 Jiyoung Jang, Min-Jeong Shin, Oh Yoen Kim, Kyong Park: Longitudinal association between egg consumption and the risk of cardiovascular disease: interaction with type 2 diabetes mellitus, in: Nutrition \& Diabetes 8 (2018) 20.

22 Richard J. Bloomer, Trint A. Gunnels, John Henry M. Schriefer: Comparison of a Restricted and Unrestricted Vegan Diet Plan with a Restricted Omnivorous Diet Plan on Health-Specific Measures, in: Healthcare (Basel) 3 (2015) 544-555.

23 http://www.sge-ssn.ch

24 Alberico L. Catapano, Ian Graham, Guy De Backer et al.: 2016 ESC/EAS Guidelines for the Management of Dyslipidaemias, in: European Heart Journal 37 (2016) 2999-3058. 
Pharmakologische Therapien, die sicher und effizient Gefässe und Herz vor den negativen Effekten von Cholesterin schützen können

Die aktuellen Leitlinien der schweizerischen Arbeitsgruppe für Lipid und Atherosklerose (AGLA) und der europäischen Gesellschaft für Kardiologie (ESC) empfehlen das Konzept der an Zielwerten orientierten Senkung des LDL-Cholesterins in Abhängigkeit von individuellen Risiken. ${ }^{25}$ Die Behandlungsindikation bei Hypercholesterinämie hängt grundsätzlich vom jeweiligen kardiovaskulären Gesamtrisiko ab, das individuell zu evaluieren ist. ${ }^{26}$

Die Statine hemmen die HMG-Co-Enzym-A-Reduktase in der Leber und damit die Cholesterinproduktion. Sie stellen die wirksamste Behandlung von erhöhtem LDL-Cholesterin dar. ${ }^{27}$ Statine sind die Therapie der ersten Wahl, da der klinische Nutzen am besten dokumentiert ist. Dabei sollte die Statin-Therapie bis zum Erreichen der LDL-Cholesterin-Zielwerte aufdosiert werden. Statine erwiesen sich bereits seit den 1990er Jahren in klinischen Endpunktstudien als wichtige Substanzklasse zur Vorbeugung von zerebro- und kardiovaskulären Ereignissen. ${ }^{28}$

Der positive Effekt von Statinen ist sowohl in der Sekundärprävention (nach einem kardiovaskulären Ereignis) als auch in der Primärprävention (bei Personen mit erhöhtem Cholesterin und noch kardiovaskulär gesund) beobachtet worden. Allerdings ist der

http:/ / www.agla.ch; A. L. Catapano, I. Graham, G. De Backer et al.: 2016 ESC/EAS Guidelines.

http:/ / www.agla.ch; A. L. Catapano usw., a.a.O.; Arnold von Eckardstein, Walter F. Riesen, David Carballo et al.: Empfehlungen zur Prävention der Atherosklerose 2018: Update der AGLA, in: Swiss Medical Forum 18 (2018) 975-980.

27 A. L. Catapano usw., a.a.O.; Jens Barthelmes, Isabella Sudano: Medikamentöse Senkung des LDL-Cholesterins, in: Praxis 106 (2017) 933940.

Cholesterol Treatment Trialists Collaboration, B. Mihaylova, J. Emberson et al.: The effects of lowering LDL cholesterol; C. Baigent, A. Keech, P. M. Kearney et al.: Efficacy and safety of cholesterol-lowering treatment. 
Effekt dieser Behandlung bei Patienten mit hohem Risiko stärker, das heisst, je höher das kardiovaskuläre Risiko ist, desto weniger Patienten müssen zur Vermeidung eines Ereignisses behandelt werden. ${ }^{29}$ Die Medikamentenklasse der Statine zeichnet sich durch hohe Sicherheit und befriedigende Verträglichkeit aus.

Häufigste Nebenwirkungen sind Veränderungen der Leberwerte und diffuse Myalgien. Die Prävalenz von Myalgien wird auf 5-10\% der Patienten geschätzt und ist der häufigste Grund für einen Therapieabbruch. Risikofaktoren für die Myalgien sind unter anderem Alter $>80$ Jahren, weibliches Geschlecht, niedriges Körpergewicht, genetische Faktoren, Intensivsport, SchilddrüsenDysfunktion, Alkoholkonsum, Konsum bestimmter Lebensmittel (Grapefruit oder Cranberry-Saft) oder Medikamente, die den Stoffwechsel der Statine beeinflussen können. ${ }^{30}$ Bemerkenswert ist, dass unter Placebo-kontrollierter Re-Exposition nach Myalgie unter Statin-Therapie 50\% der Statin-intoleranten Personen auch von Symptomen unter Placebo berichten. ${ }^{31}$

Je nach Intensität der Nebenwirkungen soll die Therapie mit Statin sistiert oder die Dosis reduziert werden. Da verschiedene Statine zur Verfügung stehen, die unterschiedliche Charakteristika aufweisen, ist zu empfehlen, verschiedene Statine auszuprobieren. Wichtig ist, dass zwischen dem Einsatz von zwei Statinen genügend Zeit liegt, so dass eventuelle Nebenwirkungen des ersten Statins verschwunden sind, bevor das zweite eingesetzt wird. ${ }^{32}$

Cholesterol Treatment Trialists Collaboration usw., a.a.O.; C. Baigent usw., a.a.O.

Erik S. Stroes, Paul D. Thompson, Alberto Corsini et al.: Statin-associated muscle symptoms: impact on statin therapy-European Atherosclerosis Society Consensus Panel Statement on Assessment, Aetiology and Management, in: European Heart Journal 36 (2015) 1012-1022.

Steven E. Nissen, Erik Stroes, Ricardo E. Dent-Acosta: Efficacy and Tolerability of Evolocumab vs Ezetimibe in Patients With Muscle-Related Statin Intolerance: The GAUSS-3 Randomized Clinical Trial in: The Journal of the American Medical Association 315 (2016) 1580-1590.

E. S. Stroes, P. D. Thompson, A. Corsini et al.: Statin-associated muscle symptoms. 
Es scheint, dass niedrig dosiertes Rosuvastatin, Fluvastatin oder Pitavastatin weniger muskuläre Symptome hervorrufen als andere Statine. ${ }^{33}$ Eine Einnahme nur jeden zweiten Tag scheint zu weniger Nebenwirkungen zu führen, aber es fehlen Daten zur langfristigen Wirksamkeit dieser Vorgehensweise. Eine Reduktion des Co-Enzyms Q10 oder von Vitamin D wurde mit erhöhter Statin-Intoleranz in Verbindung gebracht. Leider fanden die randomisierten Studien, die den Effekt von Supplementation mit dem Co-Enzym Q10 untersuchten, keine Reduktion der Myalgien durch Supplementation. Die Daten über Vitamin-D-Supplementation und Myalgien sind ebenfalls nicht überzeugend. ${ }^{34}$ Trotz des in Studien guten Sicherheitsprofils zeigt die Realität, dass im Rahmen der primären Prävention lediglich 25\% der Patienten ihr Medikament nach zwei Jahren auch weiterhin einnehmen, gegenüber $40 \%$ sekundärpräventiv nach Koronarsyndrom. ${ }^{35}$

Werden die Statine nicht toleriert oder die Zielwerte nicht erreicht, können wir andere Medikamente einsetzen. ${ }^{36}$ Ezetimibe hemmt die Cholesterinaufnahme im Darm. Die Standarddosis von $10 \mathrm{mg} / \mathrm{Tag}$ als Monotherapie senkt das LDL-Cholesterin um etwa 15-20\%. ${ }^{37}$

33 Huseyin Naci, Jasper Brugts, Tony Ades: Comparative tolerability and harms of individual statins: a study-level network meta-analysis of 246 955 participants from 135 randomized, controlled trials, in: Circulation. Cardiovascular Quality and Outcomes 6 (2013) 390-399.

A. L. Catapano, I. Graham, G. De Backer et al.: 2016 ESC/EAS Guidelines. Joseph A Hill et al.: Medical Misinformation: Vet the Message! The Journal of the American Medical Association 8 (2019) e011838; Maciej Banach, Tomáš Stulc, Ricardo Dent, Peter Paul Toth: Statin non-adherence and residual cardiovascular risk: There is need for substantial improvement, in: International Journal of Cardiology 225 (2016) 184-196; Sukyoun Shin, Sunmee Jang, Tae-Jin Lee, Ho Kim: Association between non-adherence to statin and hospitalization for cardiovascular disease and all-cause mortality in a national cohort, in: International Journal of Clinical Pharmacology and Therapeutics 52 (2014) 948-956.

36 A. L. Catapano, I. Graham, G. De Backer et al.: 2016 ESC / EAS Guidelines; J. Barthelmes, I. Sudano: Medikamentöse Senkung des LDL-Cholesterins. C. J. Packard, J. Shepherd: The pathophysiology of cholesterol metabolism in man. 
Die Kombinationstherapie mit Statinen ist besonders sinnvoll, und unter der Kombination kann das LDL-Cholesterin bis $60 \%$ gesenkt werden. Die Kombination Statin-Ezetimibe senkt das Risiko eines kardiovaskulären Ereignisses signifikant mehr als Statin allein; zudem ist die Häufigkeit von Nebenwirkungen unter StatinEzetimibe-Kombinationstherapie in etwa gleich hoch wie unter Statinen allein. ${ }^{38}$

Fibrate reduzieren in erster Linie die Triglyzeride, senken das LDL-Cholesterin um etwa 10-20\% und erhöhen leicht das HDLCholesterin. Deshalb kommt in erster Linie eine Fibrat-Therapie der mässigen bis schweren Hypertrygliceridämie in Betracht. Die Kombination von Statin mit einem Fibrat wie Gemfibrozil ist zu vermeiden, da sich das Myopathierisiko dadurch deutlich erhöht. In Gegensatz dazu dürfen Fenofibrat oder Bezafibrat zusammen mit einem Statin verabreicht werden Die Reduktion von kardiovaskulären Ereignissen durch ein Fibrat ist nur signifikant bei Patienten mit hohen Triglyzeriden. ${ }^{39}$ Anionenaustauschharze binden Gallensäuren im Darm und führen zu einer Senkung des LDLCholesterins. Die Kombination dieses Medikamentes mit Statin kann bei therapieresistenten Patienten erwogen werden. Einschränkend ist zudem die Medikationsadhärenz vermutlich aufgrund mehrmals täglicher Dosierung des unangenehm schmeckenden Pulvers und häufiger gastrointestinal unerwünschter Nebenwirkungen. ${ }^{40}$

Eine neue Therapie stellen die Inhibitoren der PCSK9 dar. ${ }^{41}$ Diese vollhumanen monoklonalen Antikörper senken die PlasmaKonzentration von PCSK9 und führen dadurch zur Erhöhung

38 Christopher P. Cannon, Michael A. Blazing, Robert P. Giugliano et al.: Ezetimibe Added to Statin Therapy after Acute Coronary Syndromes, in: The New England Journal of Medicine 372 (2015) 2387-2397.

39 http:/ / www.agla.ch; A. L. Catapano, I. Graham, G. De Backer et al.: 2016 ESC/EAS Guidelines; A. von Eckardstein, W. F. Riesen, D. Carballo et al.: Empfehlungen zur Prävention der Atherosklerose 2018.

40 http://www.agla.ch; A. L. Catapano uws., a.a.O.; A. von Eckardstein usw., a.a.O.

41 A. L. Catapano usw., a.a.O.; A. von Eckardstein usw., a.a.O.; J. Barthelmes, I. Sudano: Medikamentöse Senkung des LDL-Cholesterins. 
der LDL-Rezeptor-Dichte und somit zur Senkung des LDL-Cholesterins. Dies ist besonders wichtig für Hochrisikopatienten und Patienten mit familiärer Hypercholesterinämie, die mit anderen lipidsenkenden Therapien keine ausreichende LDL-CholesterinSenkung erreichen können. Bei Hochrisikopatienten, Patienten mit familiärer Hypercholesterinämie sowie Patienten mit StatinIntoleranz wurde gezeigt, dass diese Medikamente sehr effektiv LDL-Cholesterin senken (Reduktion um 50-70\% zusätzlich zur Statin-Wirkung und ca. 50\% als Einzeltherapie). ${ }^{42}$ Dabei werden die PCSK9-Hemmer sehr gut toleriert, und die Kombination dieser Medikamente mit Statinen senkt das Myokardinfarkt- und Hirnschlagrisiko deutlich mehr als Statin allein.

In manchen Fällen kann eine Hypercholesterinämie auch durch mehrere Medikamente nicht ausreichend gesenkt werden. Dies ist beispielsweise bei schwerer (homozygoter) familiärer Hypercholesterinämie der Fall. Bestehen zudem Gefässschäden, «wäscht» man das Blut ausserhalb des Körpers und beseitigt das zu hohe LDLCholesterin und/oder das Lipoprotein a. Anschliessend wird das nun «saubere» Blut wieder über Schläuche zum Körper zurückgeführt. Mittels der LDL-Aphärese können auch erhöhte Lipoprotein a-, IDL- und VLDL-Spiegel gesenkt werden. Das Verfahren wird für gewöhnlich einmal in der Woche durchgeführt. Parallel wird die Hypercholesterinämie weiterhin mit Medikamenten behandelt.

42 Eli M. Roth, James M. McKenney: ODYSSEY MONO: effect of alirocumab $75 \mathrm{mg}$ subcutaneously every 2 weeks as monotherapy versus ezetimibe over 24 weeks, in: Future Cardiology 11 (2015) 27-37; Michael J. Koren, Pernille Lundqvist, Michael Bolognese et al.: Anti-PCSK9 monotherapy for hypercholesterolemia: the MENDEL-2 randomized, controlled phase III clinical trial of evolocumab, in: Journal of the American College of Cardiology 63 (2014) 2531-2540. 


\section{Schlussfolgerungen}

Trotz aller Beweise, welche die Wissenschaft uns geliefert hat, hält sich das Gerücht, Cholesterin sei überhaupt kein Risikofaktor für Atherosklerose, hartnäckig. Besonders in der Laienpresse wird in regelmässigen Abständen thematisiert, dass Millionen Menschen unnötigerweise Statine einnehmen und dass Ärzte und vor allem die Pharmaindustrie Milliarden mit Cholesterinsenkern verdienen. Die Folge einer solchen Berichterstattung liegen auf der Hand: Patienten sind verunsichert und setzen ihre Medikation ab mit der Konsequenz eines erhöhten kardiovaskulären Risikos.

conexus 2 (2019) 77-89

(C) 2019 Isabella Sudano. Dieser Beitrag darf im Rahmen der Lizenz CC BY-NC-ND 4.0 - Creative Commons: Namensnennung/nicht kommerziell/keine Bearbeitungen - weiterverbreitet werden.

(c) (i) $(2)$

https: / / doi.org/10.24445/ conexus.2019.02.007

PD Dr. Isabella Sudano, Universitäres Herzzentrum Zürich, Universitätsspital Zürich, Rämistrasse 100, 8091 Zürich

isabella.sudano@usz.ch 